\title{
The Design of Multi-mode Examinees Identity Verification System for Colleges and Universities
}

\author{
Jia Chen ${ }^{1, *}$ and Cong Chen ${ }^{2}$ \\ ${ }^{1}$ Guangxi Colleges and Universities Key Laboratory of Image Processing and Intelligent Information System, Wuzhou University, \\ Wuzhou, Guangxi, China \\ ${ }^{2}$ Guangxi Colleges and Universities Key Laboratory of Professional Software Technology, Wuzhou University, Wuzhou, Guangxi, \\ China \\ ${ }^{*}$ Corresponding author
}

\begin{abstract}
With the rapid development of information management in colleges and universities, traditional eyeobserving verification methods cannot satisfy today's requirement for examination management any more. Examinees' identities need to be verified more effectively and accurately. In recent years, biometric recognition has been becoming an essential component of effective person identification solutions because biometric identifiers intrinsically represent individuals' bodily identity. In this paper, we present a multi-mode verification system that not only takes advantage of biometric recognition to improve efficiency and accuracy, but also incorporates eye-observing verification to deal with the case when biometric recognition fails. Accordingly, an original portable verification device was designed to implement this multi-mode verification system. Our experiment shows that this verification system can meet the practical requirement of examination management in colleges and universities.
\end{abstract}

Keywords-identity verification; multi-mode verification; comprehensive judgment; original verification device

\section{INTRODUCTION}

Examinee identity recognition refers to the use of distinctive anatomical and behavioral characteristics or biometric identifiers (e.g., fingerprints, face, iris, voice, hand geometry) for automatically recognizing a person who is authorized to attend the examination [1]. Examinations play an important role in selecting qualified personnel in the society, so it is critical to verify the examinees' identity accurately. Additionally, the process of verifying identity has time limitation for some examinations in colleges and universities. In practice, some people who were not authorized to take the examination were found to attend the examinations for others. Therefore, traditional verification by eye-observing cannot satisfy the requirements to verify examinees' identity in colleges and universities anymore. At present, fingerprint recognition and other identification technology have been increasingly used in some important exams such as college entrance examination in China. Examinees' fingerprints and relevant personal information were obtained when they registered for the college entrance examination. Then the examinees' identities were verified by verification devices before entering the examination room. However, the examinees identity verification systems based on biometrics haven't been commonly used in colleges and universities nowadays.
The topic of this paper is the design of a multi-mode examinees identity verification system based on face recognition and fingerprint recognition, in consideration of practical demands for colleges and universities. The structure is organized as follows: Section 2 presents the improvements for identity verification system. Section 3 gives the overview of biometrics. Then Section 4 explains the design of examinees identity verification system, and Section 5 discusses system components. Finally, Section 6 concludes the paper and points out the further work.

\section{IMPROVEMENTS FOR IDENTITY VERIFICATION SYSTEM}

\section{A. Multi-Mode Verification}

To some extent, the accuracy of biometric verification system is influenced by several objective limitations and potential errors. In order to avoid examinee misjudgment and impostors' intrusion, multi-mode verification was proposed. The verification works in the orders of face recognition, fingerprint recognition and administrator's comprehensivejudgment. The algorithm of verification is shown in Figure I. If face verification fails, fingerprints would be acquired to match the embedded fingerprint in the identification card. If the fingerprint verification fails again, the administrator will verify the examinee's identity through comprehensive information. With the help of multi-mode verification, the system can verify twins whose faces are extremely similar and the examinees with hand-related disability. Additionally, we adopt an image quality assessment method for face and fingerprint images in order to improve the verification accuracy [2]. 


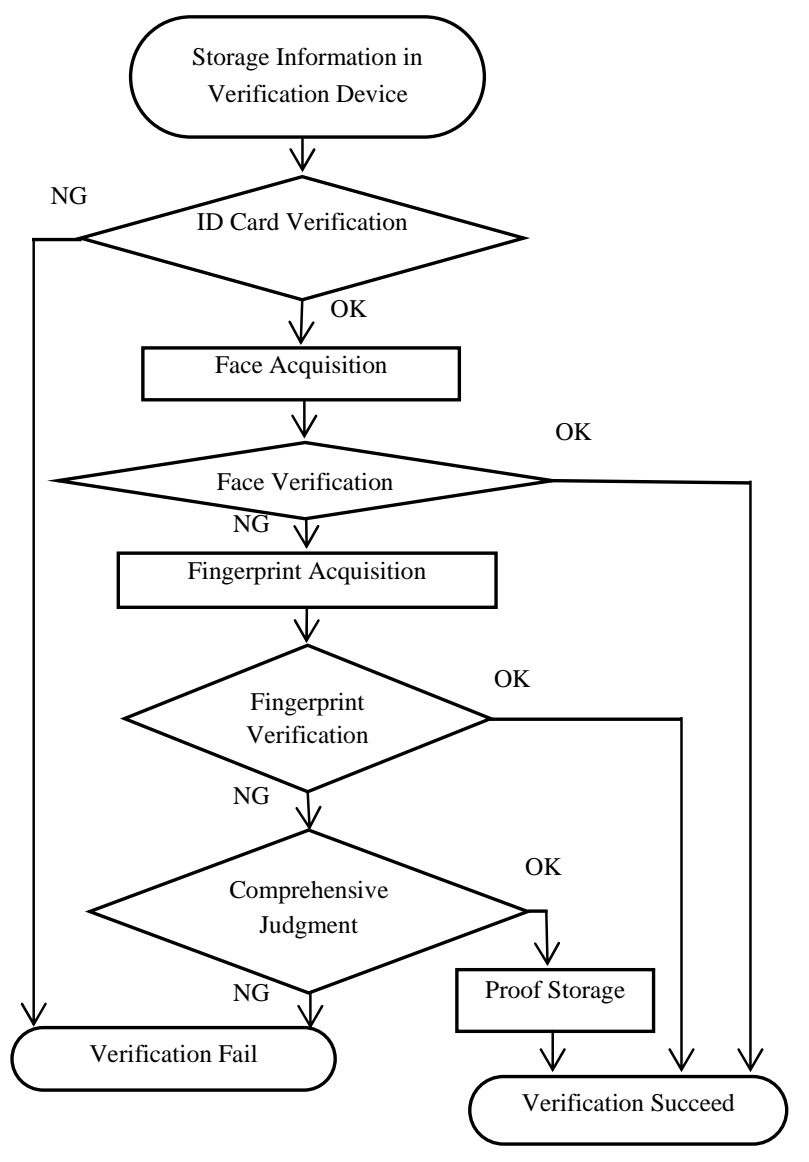

FIGURE I. PROPOSED MULTI-MODE VERIFICATION

\section{B. Original Portable Verification Device}

Thanks to introducing multi-mode verification, a portable identity verification device was proposed. The device consists of main functions as followed: identification card verification and embedded information extraction, face acquisition and verification, fingerprint acquisition and verification, specified code input for special purpose by administrator. The device can connect to network by wired or wireless ways. So the portable device can communicate with the examination management server to download or upload data.

\section{Security Improvement}

Taking into account the data security and verification reliability, the system adopts the enhanced security mechanism. The face-sample database management server and the portable verification device separately communicate with the examination management server, as shown in Figure II. The database management server also has a strict security management mechanism, so only the examination administrator is authorized to access the privileged face-sample database besides the administrator. The relevant data for each examination room is written into the memory of the device by the examination administrator. So other staff cannot contact with the data directly. Additionally, the verification device of each examination room works under the condition of standalone during the verification process without connecting to Internet or other devices, which prevents from introducing other risks for verification.

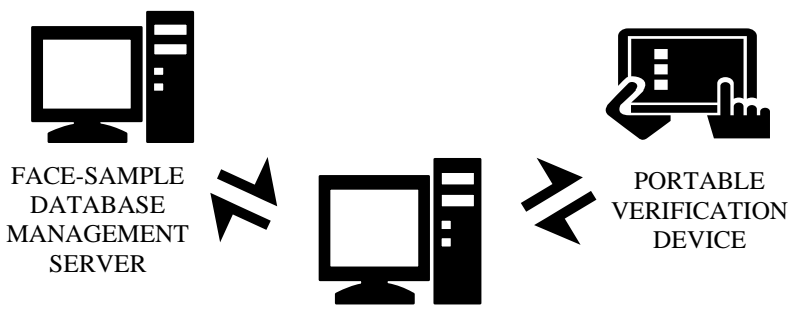

EXAMINATION MANAGEMENT SERVER

FIGURE II. PROPOSED MULTI-MODE VERIFICATION

\section{OVERVIEW OF BIOMETRICS}

Accurate automatic personal identification is becoming more and more important to our information society. Biometrics is a technology that uniquely identifies a person based on his physiological or behavioral characteristics. A number of biometric traits have been adopted in a very broad range of applications, such as faces, fingerprints, iris, voice, and hand geometry, etc. Among them, faces and fingerprints are two promising image-based biometrics [3].

Face is one of the most acceptable biometric traits and the method of acquiring face images is nonintrusive. Face recognition in images and videos has received significant attention in the recent past [4]. The different approaches in this field are classified into three categories according to the manner of treating the face image [5]. Yet, it is challenging to develop face recognition techniques that can tolerate the effects of aging, facial expression, variations in the imaging environment, and facial pose with respect to the camera.

Fingerprints are very distinctive and they are permanent; even if they temporarily change slightly due to cuts and bruises on the skin, the fingerprint reappears after the finger heals. However, fingerprint recognition also has a lot of problems: the recognition accuracy influenced by the quality of captured fingerprints and characteristics extracting algorithm, fingerprint database privacy and security, etc. In addition, the increasing fake fingerprints have brought new challenges to fingerprint recognition in recent years. The work on image quality assessment for fake biometric detection has been applied to fingerprints, face and iris in [6].

Therefore, each biometric trait has its own strengths and weaknesses, and the choice of a particular trait typically depends on the requirements of the application. A multi-mode biometric identity authentication system that based on the face, handwriting signature and iris characteristics was proposed to overcome the defects of single biological characteristics [7]. But the complexity and cost of the system increased accordingly by involving multiple biometric traits.

Taking accuracy, complexity and cost into consideration, the system combines face recognition with fingerprint recognition to verify the examinees' identity, and add a comprehensive-judgment solution to supplement biometrics recognition. 


\section{DESIGN OF EXAMINEES IDENTITY VERIFICATION SYSTEM}

\section{A. Demand Analysis}

Our verification system should verify the examinees' identity by face, fingerprint and comprehensive-judgment. This system should implement functionalities of face and fingerprint acquisition, image process, personal information input, identity verification and data management to meet the requirements of examinees identity verification, and it can improve the efficiency and accuracy.

\section{B. Overall Design of System}

The system adopts $\mathrm{C} / \mathrm{S}$ structure to design in order to ensure the data security. According to the practical process logic of examinees identity verification, the system is configured to have one administrator and one examination administrator. The administrator takes charge of routine maintenance of the system, while the examination administrator manages face database management, examinees' information management, face retrieval, examination room arrangement, examinees' information output, as well as examinees' identification results collecting.

\section{Systematic Workflow}

The identity verification system can be divided into the following parts. (1)Database Establishment and Maintenance: Face image, name, ID number and other basic information of freshmen are obtained and stored in database when students register at the enrollment department. (2)Examination management: After examinees register to attend some kind exam, their face samples and personal information will be retrieved from the database. Then the examination rooms were designated according to some rules, and the face samples and the personal information of the examinees corresponding to each examination room are exported to a specified portable verification device. (3)Identity Verification: When examinees enter the examination room, their identity will be verified one by one before entering the exam. After the authenticity of identification card is ensured, the identity will be verified by multi-mode verification. (4) Results Submit: After finishing all verification processes, the verification results and the needed proof stored by device will be submitted to the examination management server.

\section{SYSTEM COMPONENTS}

The system consists of a set of hardware and software. The hardware includes the face-sample database management server, the examination management server and the portable verification device for each examination room. Examination management server is in charge of the examinees' information management, examination room assignment, face samples extraction and output to verification device, etc. The facesample management server manages the face samples with strict access permission. The specified portable verification device is responsible for examining the authenticity of the examinees' identification card, reading the embedded information of the identification card, verifying the identity of the examinees according to the pre-stored face samples or captured fingerprints, recording the data of the verification and send results to the examination management server.

On the basis of hardware, the system software is designed with ideas of modular design ideas and provides the following main functionalities.

\section{A. Database Establishment and Maintenance}

When the students go to the colleges for registrations, their face samples and personal information are collected and input to the database. Their photos focusing on the face will be taken and it will be used for face samples after image processing. Other personal information will also be recorded, making ID number as one of the keywords.

\section{B. Examination Management}

After students register to attend the examination, the examination administrator retrieves the examinees' information from the database according to the unique ID number, including their face samples and personal information. Then the administrator organizes the examinees into different examination rooms by rules. After the examination rooms were designated, the face samples and the personal information of the examinees corresponding to each examination room are exported into a specified portable verification device through USB connection or network.

\section{Identity Verification}

As the critical function of the system, the identity verification process adopts a multi-mode identification mechanism. The verification works in the order of face recognition, fingerprint recognition and administrator's comprehensive-judgment. The examinees should take their identification cards and other certifications with them to attend the exam. When they go to the examination room, their identity will be verified one by one as the following steps.

Step1: The verification device is disconnected from the network when it begins to verify the identification. The authenticity of examinee's identification card will be examined by the verification device, and the embedded ID number and fingerprint will be extracted from the identification card and stored temporarily for use.

Step2: According to the unique ID number, the examinee's information including the face sample will be retrieved from the stored database files. The basic information (e.g. ID number, name and student ID etc.) will appear on the screen of the device. Next, the administrator staff uses the verification device to take an appropriate photo of the examinee focused on the face immediately. Then the photo will be processed by the device to match the existing face sample. The techniques used for face recognition are based on 2D face images [8]. The main steps of face recognition include face detection, feature extraction and face recognition. If the face matching process succeeds, the examinee can enter the examination room to attend the exam. Otherwise, if the face matching process fails, the process will go to step3.

Step3: The fingerprint of the examinee will be captured right now by an optical scanner embedded in the verification 
device. The fingerprint image will be processed to ensure the authenticity by a proposed algorithm [2], and then the verified image will be used to match the fingerprint sample extracted from the identification card. If the fingerprint matching process succeeds, the examinee can also enter the examination room. Otherwise, if the fingerprint matching process fails, the process will go to step4.

Step4: The administrator of the examination room will check the examinee's identity personally by comparing the photos of different identifications with the person, asking some questions, as well as searching for his/her teacher. If the administrator can make sure there is no problem with the identity, the examinee can attend the exam. Accordingly, the administrator will enter a specified code to mark the special examinee, and the photo and fingerprint captured of the examinee will be stored for reference.

Step5: After finishing all of the examinees' identification verification processes, the device will record the authentication results and other relevant data for reference. The data can be sent to the administrator server through network.

\section{Results Submit}

With the help of communication function of the device, the administrator can receive needed information from each terminal device, for example, how many examinees succeed to the pass the verification, who fails to attend the exam, and who doesn't pass the face and fingerprint verification but is acknowledged by administrator's comprehensive-judgment as well as the relevant proof, etc.

\section{CONCLUSION}

Employing the multi-mode verification method, the examinees identity verification system for colleges and universities realizes the verification through face recognition, fingerprint recognition and comprehensive-judgment. When the verification fails in one mode, the system can turn to the substitutive mode. The multi-mode verification enhances the accuracy and reliability of the system which decreases the possibility of misjudging examinees by single biometric feature. Equally important, the system will help reduce the workload of the examination administrator staff. With the help of the communication function of verification device, the examination administrator can easily collect information from each examination room in time. As a result, the system can improve the efficiency of examination management in colleges and universities. The future research work includes research on optimizing the algorithms to strengthen the fake face/fingerprint identification and decrease the image processing time, as well as learning pose-invariant face recognition [9].

\section{ACKNOWLEDGMENT}

This work was supported by the National Natural Science Foundation of China under Grant No. 61562074, Natural Science Foundation of Guangxi under Grant No. 2015GXNSFAA139295, Scientific Research of Wuzhou University under Grant No. 2011C002 and Young and MiddleAged Teacher Training Programs Fund of Wuzhou University.

\section{REFERENCES}

[1] Jain A K, Maltoni D. Handbook of Fingerprint Recognition.2nd ed., London, Springer Press, 2009, pp.2-24.

[2] Fronthaler, H., K. Kollreider, and J. Bigun. "Automatic Image Quality Assessment with Application in Biometrics." Computer Vision and Pattern Recognition Workshop, 2006. CVPRW '06. Conference on IEEE, 2006:30-30.

[3] Phillips, P. Jonathan, R. M. Mccabe, and R. Chellappa. "Biometric image processing and recognition." Signal Processing Conference IEEE, 2015.

[4] Parkhi, Omkar M., A. Vedaldi, and A. Zisserman. "Deep Face Recognition." British Machine Vision Conference 2015:41.1-41.12.

[5] M Chihaoui, A Elkefi, W Bellil and CB Amar. "A Survey of 2D Face Recognition Techniques." Computers, 2016. 5, 21.

[6] J. Galbally, Sbastien Marcel, J. Fierrez. "Image Quality Assessment for Fake Biometric Detection". in IEEE Transactions on Image Processing, Vol. 23, No. 2, Feb 2014.

[7] Guanming LU, Haibo LI, Li LIU. " A Servey on Biometrics." Journal of Nanjing University of Posts and Te lecomm unica tions(Natura l Sc ience) 27.1(2007):81-88.

[8] Hafez, Samir F., M. M. Selim, and H. H. Zayed. "2D Face Recognition System Based on Selected Gabor Filters and Linear Discriminant Analysis LDA." Computer Science 12(2015):33-41.

[9] Luan, Tran, X. Yin, and X. Liu. "Disentangled Representation Learning GAN for Pose-Invariant Face Recognition." IEEE Conference on Computer Vision and Pattern Recognition IEEE Computer Society, 2017:1283-12. 